[0212-7199 (2007) 24: 8; pp 399-403] ANALES DE MEDICINA INTERNA Copyright $(\underset{2}{ } 2007$ ARAN EDICIONES, S.L.

AN. MED. INTERNA (Madrid) Vol. 24, N. ${ }^{\circ} 8$, pp. 399-403, 2007

\title{
Papel del médico de familia en el manejo de la infección por VIH
}

\author{
L. S. BRIONGOS FIGUERO ${ }^{1,2}$, P. BACHILLER LUQUE ${ }^{2}$, J. M. EIROS BOUZA ${ }^{3}$, \\ T. PALACIOS MARTÍN ${ }^{2}$ \\ ${ }^{\prime}$ Medicina Familiar y Comunitaria. Valladolid Oeste. CS Parquesol. ${ }^{2}$ Servicio de \\ Medicina Interna. ${ }^{3}$ Servicio de Microbiología. Hospital Clínico Universitario. Valladolid
}

FAMILY PHYSICIAN'S ROLE IN THE HIV INFECTION MANAGE-
MENT

\section{RESUMEN}

La infección por el virus de la inmunodeficiencia humana (VIH) es una enfermedad con gran impacto sociosanitario. Desde 1981, cuando se describieron los primeros casos de sida, se han infectado más de 60 millones de personas. En estos 25 años se han realizado muchos avances en cuanto a su manejo y sabemos que la prevención y el diagnóstico precoz son fundamentales.

El papel del médico de familia es esencial ya que es un punto privilegiado de atención global, counseling y apoyo para estos pacientes. El principal objetivo es disminuir la incidencia de nuevas infecciones por VIH. Además, otros objetivos son: prevención primaria y promoción de la salud, diagnóstico precoz, captación de pacientes infectados, seguimiento y cuidados al paciente terminal.

Es importante conocer que todos somos susceptibles de contraer el virus y, aunque la realización de la serología de VIH es voluntaria, existen recomendaciones de los CDC: sujetos con signos o síntomas sugestivos de infección, mujeres embarazadas, sujetos con situaciones de riesgo y entre los 13 y 64 años de manera rutinaria. La comunicación del resultado es un punto clave en la relación terapéutica. Si es negativo se debe hacer intervención sobre las conductas de riesgo. Si es positivo debemos informar y apoyar al paciente, además es importante una buena captación y valoración para su derivación a la consulta especializada.

El paciente terminal de sida está muy inmunodeprimido y necesita cuidados paliativos como otra enfermedad terminal. Otro reto es la prevención y control de la infección VIH en la población inmigrante.

En conclusión, el médico de familia debe investigar prácticas de riesgo, informar, prevenir nuevos casos y, en la población infectada, seguimiento de la evolución, apoyando y confortando.

PALABRAS CLAVE: Infección por VIH/sida. Atención Primaria. Prevención.

\section{ABSTRACT}

The human immunodeficiency virus (HIV) infection is a disease with great sociosanitary impact. Since 1981, when the first cases of AIDS were described, more than 60 million people have become infected. During these 25 years there have been a lot of advances in the infection management and we know that prevention and early diagnosis are crucial.

Family Physician's role is essential since this is a privileged point of global attention, counseling and support for these patients. The main objective is to reduce the new HIV infections incidence. In addition, other objectives are: primary prevention and health promotion, early diagnosis, recruitment infected patients, monitoring and end-of-life caring.

It is important to know that all of us are susceptible to contract the virus and, although the HIV testing is voluntary, there are screening recommendations from the CDC: persons with signs or symptoms that suggest infection, pregnant women, persons at high risk for infection and all patients aged 13-64 years, as a part of routine clinical care. The communication of the result is a key point in the therapeutic relation. If it is negative we must make intervention on risk attitudes. If it is positive we must inform and support the patient, to convince him about the need to be followed up by an specialized level.

The AIDS terminal patient is a very immunodeficient one and needs palliative cares like other terminal disease. Another challenge is prevention and control of HIV infection among the immigrant community.

In conclusion, Family Physicians must investigate risk practices, inform, prevent new cases and, in the infected people, monitor the evolution, supporting and comforting.

KEY WORDS: HIV infection/AIDS. Family Physician. Prevention.

Briongos Figuero LS, Bachiller Luque P, Eiros Bouza JM, Palacios Martín T. Papel del médico de familia en el manejo de la infección por VIH. An Med Interna (Madrid) 2007; 24: 399-403.

\section{INTRODUCCIÓN}

Han pasado 25 años desde que en 1981 se publicaron los primeros casos de sida (1). En este tiempo más de 60 millones de personas se han infectado y actualmente el programa de sida de las Naciones Unidas estima que 40,3 millones de personas viven con el virus de la inmunodeficiencia humana (VIH) y que durante 2005 se registraron 4,9 millones de nuevas infecciones (2). Esto da idea del impacto de la enfermedad convirtiendo al VIH/sida en una de las principales causas de

Trabajo aceptado: 14 de marzo de 2007

Correspondencia: Laisa Socorro Briongos-Figuero. Servicio de Medicina Interna. Hospital Universitario del Río Hortega. Rondilla de Santa Teresa, 9 , 47010 Valladolid.e-mail: laisadoc@hotmail.com 
muerte en todo el mundo y en un importante problema de salud pública con gran impacto sociosanitario y diversos aspectos a tener en cuenta. Uno de ellos es que la mejora del diagnóstico precoz y el adecuado manejo, hacen que hoy día pueda considerarse una enfermedad crónica, de afectación multisistémica y multiorgánica.

Por otro lado, cabe resaltar que el diagnóstico precoz es fundamental para conseguir tanto un mayor impacto en prevención como para frenar la expansión de la infección y la aparición de diagnósticos tardíos $(3,4)$ : el 40,7\% de las personas diagnosticadas de sida en 2005 no eran conscientes de estar infectadas en el momento del diagnóstico (cifra que asciende a $63,1 \%$ entre infectados por vía heterosexual y al $62,6 \%$ entre hombres que se infectaron por medio de relaciones homosexuales) (5).

Asimismo, durante estos 25 años han sido muchos los avances realizados en cuanto al manejo general, diagnóstico y tratamiento de la infección y de los procesos concomitantes, todo lo cual supone nuevos retos y esfuerzos enmarcados en el constante proceso de actualización de los especialistas $(6,7)$.

\section{OBJETIVOS DEL MÉDICO DE FAMILIA}

El papel del médico de familia va encaminado a investigar prácticas de riesgo en relación con el VIH y asesorar sobre la infección en el contexto de la educación para la salud.

El principal objetivo desde este nivel debe ser disminuir la incidencia de nuevas infecciones fomentando conductas orientadas a promover la salud. Pero se marcan además otros objetivos $(3,8,9)$ :

-Prevención, aprovechando la continuidad y accesibilidad.

-Detección precoz.

- Captación de los pacientes infectados.

- Seguimiento en un clima agradable y empático.

-Cuidados al paciente terminal.

Para alcanzar estas metas es preciso que exista una fluida coordinación entre atención primaria y la consulta especializada, fomentando, así, una adecuada calidad de vida del paciente $(7,10)$.

\section{TRANSMISIÓN DEL VIRUS Y DEFINICIÓN DE CASO SIDA}

Es necesario conocer y recordar los mecanismos de transmisión:

- Vía parenteral: a través de sangre y derivados.

- Vía sexual: relaciones homo y heterosexuales.

- Transmisión vertical: de madre infectada a hijo durante el embarazo, parto o lactancia.

En nuestro medio, desde el inicio de la epidemia se han notificado 73.013 casos de sida. Los datos ofrecidos por el Plan Nacional sobre el SIDA muestran que el principal mecanismo de transmisión en estos casos sigue siendo el uso de drogas por vía parenteral (UDP) $(47,5 \%)$ destacando el progresivo descenso de esta vía durante los últimos años. En segundo lugar, y en continuo ascenso, se sitúa la transmisión de la infección por relaciones heterosexuales no protegidas $(30,9 \%)$. La tercera vía de transmisión más frecuente son las relaciones homosexuales entre hombres $(15,2 \%)$.
Destaca que en las mujeres la principal vía es la heterosexual $(53,9 \%)$ y en UDP existe más riesgo de seroconversión por la asociación a conductas sexuales de riesgo $(5,11)$.

La denominación de "caso sida" hace referencia a la fase avanzada (categorías 3 y C) de infección según los criterios clínicos e inmunológicos establecidos por la clasificación de los CDC (Centers for Disease Control and Prevention) de Atlanta de 1993 (Tabla I). Las categorías se describen en la tabla II $(12,13)$.

\section{TABLA I}

CLASIFICACIÓN DEL CDC DE ATLANTA DE 1993

\begin{tabular}{llcl}
\hline & \multicolumn{4}{c}{ Categorías clínicas } \\
\cline { 2 - 4 } Categoría según CD4 & $A$ & $B$ & $C^{*}$ \\
\hline 1. $>500 \mathrm{cel} / \mathrm{mm}^{3}(>29 \%)$ & A1 & B1 & C1 \\
2. $200-499 \mathrm{cel} / \mathrm{mm}^{3}(14-28 \%)$ & A2 & B2 & C2 \\
3. $<200 \mathrm{cel} / \mathrm{mm}^{3}(<14 \%)^{*}$ & A3 & B3 & C3 \\
\hline
\end{tabular}

${ }^{*}=$ casos sida
PAPEL DE ATENCIÓN PRIMARIA: PREVENCIÓN DE LA INFECCIÓN

La infección se presenta con gran repercusión sociosanitaria, económica y ética, afectando al individuo a todos los niveles, a las familias, la comunidad y la salud pública $(4,14)$.

Se sabe que la prevención es la medida más útil para paliar el avance del VIH y romper la cadena epidemiológica, por tanto, la actividad realizada en atención primaria puede llegar a ser un lugar privilegiado de prevención primaria y secundaria por estar integrada en el seno de la comunidad y en sus actividades $(3,6,8,15)$.

En este punto cabe destacar la efectividad del consejo: los especialistas adquieren durante su formación habilidades y experiencia en la atención de diversas patologías y también de la infección por el VIH. Así, el counseling o asesoramiento, junto con técnicas de entrevista y el diagnóstico precoz, se ha convertido en un punto clave a la hora de dar apoyo a los infectados por el virus y prevenir nuevas infecciones $(6,16-$ $18)$.

\section{SEROLOGÍA, ¿A QUIÉN?}

Es importante saber que todos somos susceptibles de contraer el virus ya que es una patología asociada a conductas y comportamientos $(3,19)$. Pero esta afirmación no es una condición para la realización de serologías a todos sino que debería ofrecerse y realizarse de manera rutinaria a toda la población entre 13 y 64 años y especialmente a $(20,21)$ :

- Todo aquel que lo solicite.

- A las embarazadas.

-Pero, sobre todo, en:

- Sujetos con prácticas o situaciones de alto riesgo (Tabla III). En este grupo es importante la repetición al menos anual de la serología.

- Sujetos con clínica sugestiva de infección (Tabla IV).

- Pacientes diagnosticados de enfermedad sugerente de infección por VIH (Tabla IV). 
TABLA II

DESCRIPCIÓN DE LAS CATEGORÍAS CLÍNICAS EN ADULTOS, SEGÚN LOS CDC

\section{Categoría A}

- Pacientes asintomáticos

- Linfadenopatía persistente generalizada en dos o más localizaciones extrainguinales más de tres meses o infección aguda por VIH

- Infección aguda por VIH

\section{Categoría $B$}

- Angiomatosis bacilar

- Candidiasis oral (muguet)

- Candidiasis vulvovaginal persistente, frecuente o que responde mal al tratamiento

- Displasia cervical moderada-severa o carcinoma in situ

- Temperatura superior de $38,5^{\circ} \mathrm{C}$ y diarrea más de un mes

- Leucoplasia oral vellosa

- Herpes zoster (dos episodios o uno que afecte a más de un dermatoma)

- Púrpura trombocitopénica idiopática

- Listeriosis

- Enfermedad inflamatoria pélvica

- Neuropatía periférica
Categoría C

- Candidiasis traqueal, bronquial, pulmonar o esofágica

- Criptococosis extrapulmonar

- Criptosporidiasis o isosporidiasis con diarrea más de un mes

- Infección por CMV en el niño de más de un mes de edad (en localización distinta a hígado, bazo o ganglios linfáticos

- Retinitis por CMV

- Encefalopatía por VIH

- Herpes simple que causa una úlcera cutánea de más de un mes de evolución

- Histoplasmosis diseminada (en una localización diferente o además de los pulmones, ganglios cervicales o hiliares)

- Sarcoma de Kaposi

- Linfoma de Burkitt o equivalente

- Linfoma inmunoblástico o equivalente

- Linfoma cerebral primario o equivalente

- Tuberculosis pulmonar, extrapulmonar o diseminada

- Infección por M. avium complex o M. kansasii diseminada o extrapulmonar

- Infección por otras micobacterias extrapulmonar o diseminada

- Neumonía por $P$. jiroveci

- Neumonía recurrente (más de 2 episodios/año)

- Leucoencefalopatía multifocal progresiva

- Sepsis recurrente por Salmonella sp. diferente a S. typhi

- Toxoplasmosis cerebral

- Síndrome caquéctico (Wasting syndrome)

- Carcinoma de cérvix invasivo

- Coccidiomicosis diseminada (en una localización diferente o además de la pulmonar o los ganglios linfáticos cervicales o hiliares)

\section{TABLA III}

\section{PRÁCTICAS Y/O SITUACIONES DE RIESGO}

Usuarios de drogas intravenosas

Promiscuidad sexual

Contacto con prostitutas

Enfermedades de transmisión sexual, hepatitis por virus $B$ y/o hepatitis por virus $C$ en los últimos 25-30 años

Pinchazo accidental con material contaminado

Transfusiones y trasplantes antes de 1987

Profesionales expuestos

El embarazo y la lactancia

Hijos de madre VIH

Pareja estable infectada o con prácticas y/o situaciones de riesgo

La realización de la prueba es voluntaria y, siguiendo las últimas recomendaciones de los CDC, no es necesario un consentimiento específico firmado ya que la aceptación general de los cuidados médicos debería considerarse suficiente (20).

\section{COMUNICACIÓN DEL RESULTADO}

Es uno de los momentos clave en la relación terapéutica.

\section{TABLA IV}

MANIFESTACIONES SUGESTIVAS DE INFECCIÓN POR EL VIH

Síndrome "mononucleosis-like" (con o sin exantema maculopapular, que es la manifestación cutánea más precoz de la infección por VIH)

Síndrome poliadenopático

Fiebre de origen desconocido

Adelgazamiento injustificado

Citopenias (trombopenias) crónicas

Diarrea crónica

Molluscum contagiosum

Dermatitis seborreica

Muguet de repetición

Cualquier enfermedad de transmisión sexual

Neumonía neumocócica o por $H$. influenzae o neumonía recurrente en paciente joven

Tuberculosis

Síndrome constitucional persistente

Es necesario crear un clima agradable y empático para no condicionar la comunicación con el paciente. El resultado debe darse al paciente en persona (nunca por teléfono), con tiempo suficiente, si es posible en consulta programada al principio de la semana para prever una segunda visita en un corto periodo de tiempo. 
TABLA V

PAUTAS VACUNALES EN EL PACIENTE VIH

\begin{tabular}{ll}
\hline Hepatitis B & $\begin{array}{c}\text { Usar doble dosis. Realizar un control tras la } 3^{\text {a }} \text { dosis y si no hay respuesta adminis- } \\
\text { trar una } 4^{\mathrm{a}} \text { e incluso una } 5^{\mathrm{a}} \text { dosis }\end{array}$ \\
\hline Neumococo & Revacunación cada 5 años \\
\hline Gripe & Administrar anualmente a los pacientes VIH y contactos próximos \\
\hline Tétanos-difteria & Dosis de recuerdo cada 10 años \\
\hline H. influenzae & No recomendada ya que la mayoría de las infecciones son por cepas no tipables \\
\hline Hepatitis A & $\begin{array}{c}\text { Grupos de alto riesgo (UDI, viajeros a zonas endémicas, personas con hepatitis C } \\
\text { crónica y homosexuales activos) }\end{array}$ \\
\hline Sarampión-rubéola-parotiditis & Misma pauta que en la población adulta \\
\hline
\end{tabular}

Consideraciones: Evitar las vacunas con virus vivos (BCG, polio oral, fiebre amarilla, tifoidea oral) salvo la triple vírica. Las vacunaciones pueden causar aumento transitorio de la carga viral, por ello, determinación distante 4 semanas de la administración de la vacuna. Si recuento de linfocitos CD4 < $200 / \mathrm{mm}^{3}$ valorar terapia antirretroviral.

Si el resultado es negativo debemos aprovechar la situación para realizar intervención sobre las conductas de riesgo e intentar modificarlas programando visitas con el paciente y negociando cambios de hábitos.

Cuando el resultado es positivo es preciso informar de forma clara y asertiva y apoyar al paciente. El principal objetivo de este encuentro debe ser comunicar el estado de positividad, valorar el impacto emocional y atender a las demandas inmediatas que puedan surgir. Un acompañamiento no verbal empático, asegurando la confidencialidad y con actitud de escucha activa, puede ser mucho más útil para favorecer la descarga emocional $(3,6,19,22)$.

\section{LA CAPTACIÓN DEL PACIENTE}

Al encontrar un resultado positivo debemos realizar captación de estos pacientes y derivación de todos ellos a la consulta especializada, pero valorando el momento de la infección y el estado inmunológico en el que se encuentra el paciente mediante $(3,19,22)$ :

-Anamnesis: investigar, en el paciente y en su pareja/s, las prácticas y situaciones de riesgo(historia sexual, drogas); la fecha probable de infección; las vacunaciones recibidas y las infecciones pasadas, como tuberculosis o hepatitis; identificar síntomas actuales asociados a infección por VIH y evaluar la calidad de vida del paciente y su soporte psicosocial.

-Exploración física: valoración del estado general y nutricional y, especialmente, piel y mucosas con atención a la cavidad oral y ganglios linfáticos.

- Pruebas complementarias que orientan sobre el estado inmunológico del paciente:

- Hemograma (importantes la fórmula leucocitaria y el recuento plaquetario), velocidad de sedimentación (VSG), coagulación, bioquímica (valorar función renal, hepática y perfil lipídico), sedimento de orina (valora nefrotoxicidad medicamentosa, glomerulopatías e infecciones).

- Proteinograma, albúmina y prealbúmina, vitamina B12, ácido fólico.

- Serología de hepatitis B y C, lúes, toxoplasma, citomegalovirus, prueba de mantoux.
- Poblaciones linfocitarias y carga viral.

- Radiografía de tórax y ECG que sirvan de referencia.

- Citología cervicovaginal.

\section{SEGUIMIENTO}

Aprovechando la continuidad en las visitas al médico de familia, se puede hacer un buen seguimiento del paciente y elaborar un plan de cuidados en colaboración con el propio paciente reforzando las medidas preventivas para alcanzar una buena calidad de vida (19,23-25):

- Medidas higiénico dietéticas: mantener 8 horas de sueño, realizar ejercicio físico según las capacidades individuales, mantener la actividad laboral, realizar una dieta adecuada con buen soporte nutricional.

- Abordar el estado emocional del paciente, sus preocupaciones y sus miedos.

- Mantener y potenciar las relaciones sociales, sin "obligar" a aumentarlas.

- Aconsejar sobre conductas de vida saludables.

-Ofrecer ayuda y poner en contacto con los recursos sociales disponibles.

- Abordar el miedo al rechazo dentro de su ambiente habitual y ayudar a comunicar su situación a sus contactos, resolución de dudas en la convivencia diaria.

- Vigilar los signos de progresión de la enfermedad.

- Prevención, detección precoz y tratamiento de las infecciones y tumores oportunistas.

- Recomendar pautas vacunales (Tabla V).

- Potenciar la adherencia al tratamiento antirretroviral y quimioprofiláctico, identificar los efectos secundarios y las interacciones con otros medicamentos.

\section{CUANDO SE ACERCA EL FINAL}

El paciente terminal de sida está muy inmunodeprimido con patologías como ceguera y demencia y que requiere cuidados paliativos como cualquier otra enfermedad terminal, sabiendo que se debe mantener la profilaxis y, en el caso de heroinómanos, el tratamiento sustitutivo con metadona. Es 
importante que antes se hable de ello sin barreras, para facilitar al paciente una fluida comunicación y la libre expresión de sus sentimientos hacia el momento del final de su vida $(15,26)$.

\section{NUEVOS RETOS}

Los que se ven abocados a la inmigración son en su mayoría personas sanas, pero pueden proceder de países con alta prevalencia de infección por el $\mathrm{VIH} /$ sida o haber estado expuestas a situaciones de riesgo durante su viaje. La inmigración va ligada a novedades importantes en la vida de las personas, pudiendo presentar una situación de inestabilidad. Así, nos encontramos con un aumento de diagnósticos de la infección en población inmigrante, grupo sobre el cual la idea de la prevención es conseguir que sean capaces de identificar con qué prácticas se arriesgan a infectarse y a infectar a otros y que adopten medidas preventivas que les protejan de dicha exposición $(3,5,7,27)$.

\section{CONCLUSIÓN}

El médico de familia debe investigar las prácticas de riesgo con respecto al VIH y asesorar e informar sobre la infección, para prevenir nuevos casos, fomentando hábitos de vida saludables.

En la población infectada, la actuación desde atención primaria debe dirigirse no sólo a la detección precoz, sino también a la instauración y vigilancia de medidas preventivas de reducción de riesgo de transmisión a terceros y adquisición de enfermedades concomitantes dentro del control, seguimiento y colaboración con la consulta especializada, asesorando a nuestros pacientes durante su evolución, sin juzgar, simplemente ayudando y confortando.

\section{Bibliografía}

1. Pneumocystis pneumonia. Los Angeles. MMWR Morb Mortal Wkly Rep 1981; 30: 250-2.

2. Situación de la epidemia de sida, diciembre de 2005. En internet: http://www.unaids.org

3. Aguado Taberna C, Fransi Galiana L. Virus de la inmunodeficiencia humana. AMF 2006; 2: 260-9.

4. Castilla J, Noguer I, Belza MJ, del Amo J, Sánchez F, Guerra L. ¿Estamos diagnosticando a tiempo a las personas infectadas por el VIH? Aten Primaria 2002; 29: 20-5.

5. Vigilancia epidemiológica del sida en España. Registro Nacional de Casos de Sida. Acualización a 30 de Junio de 2006. Informe Semestral nº1, año 2006. En internet: http://www.isciii.es/htdocs/centros/epidemiologia/epi_sida.jsp

6. Khalsa AM. Preventive counseling, screening, and therapy for the patient with newly diagnosed HIV infection. Am Fam Physician 2006; 73: $271-80$.

7. Thomas Mulet V, Aguado Taberna C, Fidalgo González S, Fransi Galiana L, Gutiérrez Jodra M, Mascort Roca J, et al. La infección por el VIH/sida y atención primaria. Aten Primaria 2004; 33: 3-5.

8. Hecht FM, Wilson IB, Wu AW, Cook RL, Turner BJ. Optimizing care for persons with HIV infection. Society of General Internal Medicine AIDS Task Force. Ann Intern Med 1999; 131: 136-43.

9. Coates TJ. HIV prevention in clinical practice. AIDS Clin Care 1999; 11: 1-2, 8 .

10. Guenter D, Scott S. Short report: Canadian family doctors caring for people with HIV and AIDS. Canada's National Family Physician Workforce Survey. Can Fam Physician 2004; 50: 1011-3.

11. Situación hospitalaria de pacientes VIH/sida. Resultados 2004. Análisis de la evolución 1996-2004. En internet: http://www.isciii.es/htdocs/ centros/epidemiologia/epi_sida.jsp

12. CDC. 1993 Revised Classification System for HIV Infection and Expanded Surveillance Case Definition for AIDS Among Adolescents and Adults. MMWR Recomm Rep 1992; 41 (RR-17): 1-19.

13. CDC. 1993 Revised Classification System for HIV Infection and Expanded Surveillance Case Definition for AIDS Among Adolescents and Adults. MMWR Morb Mortal Wkly Rep 1992; 41: 961-2.

14. Da Pena JM. Papel del médico de atención primaria en el sida. Aten Primaria 1996; 18: 103-4.

15. Chamorro J, Valpuesta Y, Eiros JM, Moreno L, Gonzalez I, Martin C, et al. Actitud del personal sanitario de atencion primaria ante la infec- cion VIH. Resultados de una encuesta. Aten Primaria 1994; 13: 484-6.

16. Feldman J, Miner M, Millis M. Training family practice residents in HIV care. AIDS Patient Care STDS 2004; 18: 395-404.

17. Haidet P, Stone DA, Taylor WC, Makadon HJ. When risk is low: primary care physicians' counseling about HIV prevention. Patient Educ Couns 2002; 46: 21-9.

18. Casabella Abril B, Llobera Serentill A, Pérez Sánchez J, Sala Álvarez C, García Ortega D. Actitudes y necesidades de formación sobre la infección por el VIH en profesionales sanitarios de atención primaria. Aten Primaria 1997; 19: 395-9.

19. Guerra Romero L, Sánchez de Vega T. Prevención y asistencia de la infección por VIH en atención primaria.Secretaría del plan nacional sobre el sida. Ministerio de sanidad y consumo; 2001.

20. CDC. Revised recommendations for HIV testing of adults, adolescents, and pregnant women in health-care settings. MMWR Recomm Rep 2006; 55 (RR-14): 1-17.

21. Bayer R, Fairchild AL. Changing the Paradigm for HIV Testing. The end of exceptionalism. N Engl J Med 2006; 355: 647-9.

22. Epstein RM, Morse DS, Frankel RM, Frarey L, Anderson K, Beckman HB. Awkward moments in patient-physician communication about HIV risk. Ann Intern Med 1998; 128: 435-42.

23. Guidelines for the Use of Antiretroviral Agents in HIV-1-Infected Adults and Adolescents.October 10, 2006. Developed by the Panel on Clinical Practices for Treatment of HIV infection convened by the Department of Health and Human Services (DHHS). En internet: http://AIDSinfo.nih.gov

24. Knobel H, Escobar I, Polo R, Ortega L, Martin-Conde MT, Casado JL, et al. Recomendaciones GESIDA/SEFH/PNS para mejorar la adherencia al tratamiento antirretroviral en el año 2004. Enferm Infecc Microbiol Clin 2005; 23: 221-31.

25. CDC. Updated U.S. Public Health Service guidelines for the management of occupational exposures to HIV and recommendations for postexposure prophylaxis. MMWR Recomm Rep 2005; 54 (RR-9): 1-17.

26. Curtis JR, Patrick DL, Caldwell ES, Collier AC. Why don't patients and physicians talk about end-of-life care? Barriers to communication for patients with acquired immunodeficiency syndrome and their primary care clinicians. Arch Intern Med 2000; 160: 1690-6.

27. Perales-Fraile I, Ramos-Martínez A, Asensio-Vegas A, Martín-Jiménez T. Características de la infección por VIH en la población inmigrante. Enferm Infecc Microbiol Clin 2006; 24: 407-8. 